\title{
MEROMORPHIC SOLUTIONS OF LINEAR DIFFERENTIAL SYSTEMS, PAINLEVÉ TYPE FUNCTIONS
}

\author{
LEV SAKHNOVICH
}

Abstract. We consider the $n \times n$ matrix linear differential systems in the complex plane. We find necessary and sufficient conditions under which these systems have meromorphic fundamental solutions. Using the operator identity method we construct a set of systems which have meromorphic solutions. We prove that the well known operator with the sine kernel generates a class of meromorphic Painlevé type functions. The fifth Painlevé function belongs to this class. Hence we obtain a new and simple proof that the fifth Painlevé function is meromorphic.

Mathematics subject classification (2000): 34M05, 34M55, 47B38.

Key words and phrases: Strong regularity, global solution, meromorphic solution, differential system with parameter, operator identity method, Painlevé type functions, elliptic functions.

\section{REFERENCES}

[1] N. I. AKHIEZER, Elements of the Theory of Elliptic Functions, Amer. Math. Soc., Providence, 1990.

[2] L. BIEBERBACH, Theorie der Gewönliche Differentialgleichungen, Springer-Verlag, Berlin, 1965.

[3] A. ChERVOV AND D. TAlalAEV, KZ equation, G-opers and quantum Drinfeld-Sokolov reduction, arXiv:hep-th/0607250, 2006.

[4] E. A. Coddington And N. Levinson, Theory of Ordinary Differential Equations, McGraw-Hill Book Company, New York, 1955.

[5] C. F. Coleman and J. R. Mclaughlin, Solution of the Inverse Spectral Problem for an impedance with integrable derivative, Comm. Pure Appl. Math., 56 (1993), 145-184.

[6] P. A. DeIfT, A. R. Its AND X. ZHOU, A Riemann-Hilbert Approach to Asymptotic Problems Arising in the Theory of Random Matrix Models, and also in the Theory of Integrable Statistical Mechanics, Annals of Math., 146 (1997), 149-235.

[7] H. Dym And L. SAKHNOvich, On dual Canonical Systems and Dual Matrix String Equations, Operator Theory, 123 (1997), 207-228.

[8] F. Gesztesy and W. Stcka, On Theorem of Picard, Proceeding of the Amer. Math. Soc., 126 (1998), No. 4, 1089-1099.

[9] I. GoHBerg AND M. G. KREIN, Theory and Applications of Volterra Operators in Hilbert Space, Amer. Math. Soc. Providence, 1970.

[10] V. I. Gromak, I. Laine And S. Shimomura, Painlevé Differential Equations in the Complex Plane, de Gruyter Studies in Mathematics, 28 (2002), Berlin, New York.

[11] I. S. KaC And M. G. KREIN, On the Spectral Function of the String, Amer. Math. Soc. Translation, 103 (1974),1-18.

[12] E. KAMKE, Differentialgleichungen: Losungsmethoden und Losungen, Bd. 2, Teubner, Germany, 1983.

[13] T. KATO, Perturbation Theory for Linear Operators, Springer-Verlag, Berlin, 1976.

[14] V. KATSNELSON AND D. VOLOK, Rational Solutions of the Schlesinger System and Isoprincipal Deformations of Rational Matrix Functions II. Operator Theory: Advances ana Applications, 157 (2005), 165-203.

[15] M. G. KreIN, On Main Approximation Problem of Extrapolation Theory and Filtration of Stationary Stochastic Processes, Dokl. Akad. Nauk SSSR, 94 (1954), No. 1, 13-16.

[16] M. L. MeHTA, Random Matrices, Academic press, Boston, 1991. 
[17] J. Rovnyak And L. A. SAKhnovich, Inverse Problems for Canonical Differential Systems with Singulariries, Operator Theory: Advances and Applications, to appear.

[18] L. A. SAKHNOVICH, Factorization of Operators in $L^{2}(a, b)$, Functional Anal. Appl., 13 (1979), 187-192 (Russian).

[19] L. A. SAKHNOVICH, Spectral Theory of Canonical Differential Systems. Method of Operator Identities, 107, Operator Theory Advances and Appl., Birkhäuser, 1999.

[20] L. A. SAKHNovich, On Reducing the Canonical System to the Two Dual Differential Systems, J. Math. Anal. Appl., 255 (2001), No. 2, 499-509.

[21] L. A. SAKHNOvich, Rational solutions of Knizhnik-Zamolodchikov system, arXiv:math-ph/0609067, 2006.

[22] C. A. TRACY AND H. WIDOM, Introduction to Random Matrices, Springer Lecture Notes in Physics, $\mathbf{4 2 4}$ (1993), 103-130.

[23] C. A. Tracy and H. Widom, Level spacing distribution and the Bessel kernel, Common. Math. Phys., 161 (1994), 289-309.

[24] C. A. TRacy And H. Widom, Level spacing distribution and the Airy kernel, Common. Math. Phys., 159 (1994), 151-174.

[25] W. WASOw, Asymptotic Expansions for Ordinary Differential Equations, Pure and Appl. Math., 14 (1965). 